\title{
PROBLEMATIC ALCOHOL USE IN TEAM AND INDIVIDUAL EXERCISE SETTINGS
}

\author{
Rikke Aarhus Høglid, Attila Szabo \\ ELTE Eötvös Loránd University, Budapest, Hungary
}

\begin{abstract}
Background. The scholastic literature suggests that alcohol use may be a problem in team sports, but data on the general or average exercisers are lacking. This inquiry examined the prevalence of use and the level of problematic use of alcohol in a highly heterogeneous group of team and individual exercisers.

Methods. Two-hundred exercising male $(n=79)$ and female $(n=121)$ participants $(M$ age $=26.79 \pm S D=7.88$ years) answered demographic questions and completed the Alcohol Use Disorder Identification Test (AUDIT).

Results. The rate of alcohol drinkers did not differ between the groups, but males exercising in team settings reported higher levels of alcohol drinking problems than individual exercisers in general. The findings were similar for older (aged 25 years and over) adults too, as shown by a separate analysis of the latter group. The frequency and volume of exercise were unrelated to problematic alcohol use. Cases of high level of alcohol use problems were twice as many in team than in individual exercisers.

Conclusions. The prevalence of alcohol use in team and individual exercisers is similar, but problematic alcohol use is greater in the former group. Increased alcohol use problems in team exercisers seem to be limited to men and it occurs in older adults too. Men drink more than women, but the behaviour may have a general social, rather than a sport-specific, context.
\end{abstract}

Keywords: dependence, drinking, physical activity, social, substance use.

\section{INTRODUCTION}

I n their literature review of youngsters under 25 years of age, Lisha and Sussman (2010) observed that 22 studies out of the 34 examined found that sport participants reported higher levels of drinking than non-participants. A systematic review of longitudinal studies also showed that in 15 out of the 19 reviewed studies alcohol use was positively associated with sports participation in adolescents and young adults (Kwan, Bobko, Faulkner, Donnelly, \& Cairney, 2014). A study with young men, aged 16 to 34 years who took part in non-elite sports, showed that excessive drinking was more likely with teammates than with other social groups (Black, Lawson, \& Fleishman, 1999). One longitudinal study revealed that participation in team sports was related to increased alcohol use problems in the late adolescence and early adulthood (T. Wichstrøm \& L. Wichstrøm, 2009). Finally, studying a sample of high school athletes, Kulesza Grossbard, Kilmer, Copeland, and Larimer (2014) found that team-sport participation was associated with greater alcohol related problems than participation in individual sports. The scholastic literature provides convincing evidence that youngsters involved in team sports might be more susceptible to drinking problems than their counterparts involved in individual sports.

There are some unresolved questions, however, concerning the link between team and individual forms of exercises and alcohol use and misuse. One is whether the results obtained in studies with athletes can be extrapolated to the average exercisers. Further, since most studies were with young athletes in specific sports, could these 
finding be applied to a heterogeneous, or the general exercising population who perform a very wide spectrum of activities with different frequencies and intensities? As the difference in alcohol related problems between team and individual sport participants is not consistent across sports (Sønderlund et al., 2014), one point that begs for clarification is whether the unique social context of a very specific sport, or simply the general social environment is what makes people more vulnerable to alcohol misuse. Research shows that outside the domain of sports and exercise, socialization and selection are both determinants of heavy drinking (Becker \& Curry, 2013). Therefore, it is plausible that team physical activity, whether it is exercise for fun or competitive sport, is a stage that provides opportunities for both selection and socialisation, in which case certain personal motivational factors gravitate people towards team or individual sports; then the link between taking part in sports and alcohol use problems may need to be re-evaluated.

In this inquiry we looked at a sample of randomly generated heterogeneous exercisers, participating in over 25 different sports, representing both genders, with a wide age range (62 years), wide frequency-range of weekly workouts (16 times), and wide range of total weekly hours of exercise (69 hours). This sample composition is different from the specific athletes and very young samples studied to date. Based on the non-sport link between alcohol use and socialization (Becker \& Curry, 2013), we hypothesized that alcohol use problems might surface in team exercisers, in general, or regardless of the level of involvement, weekly frequency or intensity of exercise, simply because of the social context in team physical activities. To test our hypothesis, we compared a randomly generated sample of very heterogeneous (on purpose) team and individual exercisers on the basis: (i) of the prevalence of alcohol use and (ii) based on the level of problematic alcohol use. It was our aim to answer the question whether participation in physical activities, in general, could be associated with different drinking patterns in team and individual exercisers.

\section{METHODS}

Participants. The current research was conducted with ethical approval of the Research Ethics Committee of the Faculty of Education and Psychology at Eötvös Loránd University in
Budapest, Hungary. Participants were recruited for another study testing the co-occurrence of substance and exercise addiction (Szabo, Griffiths, Aarhus Høglid, \& Demetrovics, 2018). They were randomly selected from a pool of 691 people who completed the survey on condition that they participate in individual or team exercises. The selection was based on a priori sample size calculation using the G*Power (v. 3) software (Faul, Erdfelder, Buchner, \& Lang, 2009). This test indicated that the minimum required sample size was 172 (as based on: $f=.25, \alpha=.05$, and $1-\beta=$ 90). This number was increased to 200 in order to work with equal sample sizes (100 individual and 100 team-exercisers). The random sample was generated with the select random cases feature of the SPSS software, which resulted in 79 men and 121 women who were included in the final sample. Participants' mean $(M)$ age was $26.79 \pm S D=7.88$ years, they exercised $4.45 \pm S D=2.21$ times per week for an average of $2.06 \pm S D=6.28$ hours per workout. Most of the current sample were Scandinavians $(77.2 \%)$, while the rest were from other European countries (22.5\%).

Materials. Apart from the demographic questions, participants performed the 10-item Alcohol Use Disorders Identification Test (AUDIT; Babor, Higgins-Biddle, Saunders, \& Monteiro, 2001) to assess problematic drinking among those who answered "yes" $(n=162 / 200)$ to alcohol consumption within the past year. Cut-off scores are: $0-7$ reflecting low risk, $8-15$ reflecting medium risk, or hazardous drinking, and a score of 16 or above reflects a high risk, or a high level of alcohol use problems. In the current inquiry, the internal consistency of the scale was acceptable $(n=200$; $\alpha=.75$ ).

Procedure and statistical analyses. The randomly generated data of 200 team and individual exercise participants were subjected to statistical analysis using the SPSS software. There were two specific questions addressed in this work: 1) Is there a greater prevalence of alcohol use among team than individual exercisers? and 2) Is problematic alcohol use (as based on the AUDIT scores) greater in team than in individual exercisers? This work was based on a betweenparticipants design calling for an independent $t$-test if the assumptions for a parametric test are met. Thus, first we adopted the Kolmogorov-Smirnov and Shapiro-Wilk tests to verify the assumption of normality. Since the latter was not met, we 
used the Mann-Whitney $U$-test, which is a nonparametric alternative to independent $t$-test. For examining the frequency of alcohol users in team and individual sports, we used the chi-square test. Finally, Spearman's rho $(\rho)$ correlations were used to determine the relationship between AUDIT scores and the weekly frequency and the weekly volume (hours) of exercise. The level of statistical significance was set to .05 for all tests.

\section{RESULTS}

The first question was examined with a Chisquare, which yielded no statistically significant difference between the prevalence of alcohol use in team $(88 / 100)$ versus the individual $(87 / 100)$ exercisers.

Regarding the second question, since the Kolmogorov-Smirnov and Shapiro-Wilk tests showed that the assumption of normality was violated in the data $(p<.001)$, a Mann-Whitney $U$ test was used to determine if problematic alcohol use was greater among team than individual exercisers. This test revealed that team exercisers $(M d n=7, M$ rank $=96.82)$ differed statistically significantly from individual exercisers $(M d n=6$, $M$ rank = 79.08) in their total AUDIT scores $(U=$ $-2.307, p=.02)$. Based on these scores, participants were categorized as having low $(<8)$, medium $(8-$ $15)$, and high levels $(>15)$ of alcohol use problem (Babor et al., 2001). There were twice as many (6/82 (7.3\%), 5/1 men/women ratio) versus $3 / 80$ (3.8\%) $1 / 2$ men/women ratio) high level alcohol use problems among team exercisers than individual exercisers, and two-thirds (4/6) of the team exercisers reached the cut-off score of 20 (all men), which calls for the clinical evaluation of alcohol dependence (Babor et al., 2001). None of the AUDIT scores reached 20 or more among individual exercisers (Figure). Spearman's rho $(\rho)$ correlations indicated that the AUDIT scores were unrelated to the weekly frequency or weekly volume (hours) of exercise, but they were negatively related to the age of the participants $(\rho=-20, p=.01)$.

In the subsequent analyses, we compared the AUDIT scores of men $(n=61$; mean rank $=95.50)$ with those of women $(n=101$; mean rank $=73.04)$, which were statistically significantly different (Mann-Whitney $U=-2.966, p=.003$ ) and led us to re-run the initial analyses for team versus individual exercisers, separately for males and females. These tests appeared to be statistically significant only for men $(U=-2.204, p=.028)$, revealing that males involved in team exercises $(n=35 ; M$ rank $=35.30)$ scored higher on the AUDIT than those practicing individual forms of exercises $(n=26 ; M$ rank = 25.21). Finally, due to the statistically significant correlation between the AUDIT scores and age, as well as because earlier studies primarily examined adolescents or young adults, we re-ran the test for those aged 25 and over $(n=67)$, which again resulted in a statistically significant $(U=-2.317, p=.021)$ difference between team $(n=32 ; M$ rank $=39.73)$ and individual exercisers $(n=35 ; M$ rank $=28.76)$, showing that the former had higher AUDIT scores than the latter.

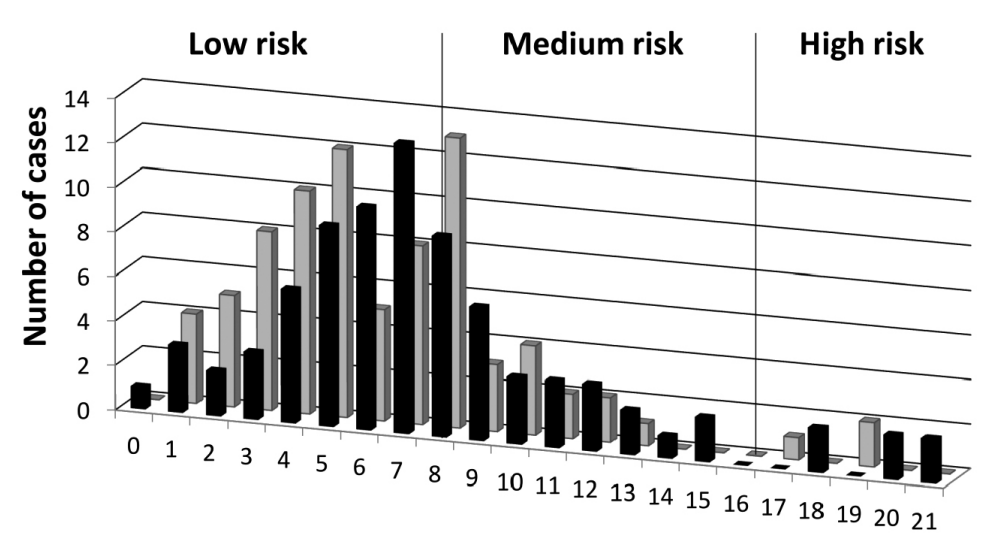

Total AUDIT scores (low risk < 8, medium risk 8-15, high risk > 15)

- Team $(\mathrm{n}=82) \quad \square$ Individual $(\mathrm{n}=80)$
Figure. Distribution of the scores obtained on the Alcohol Use Disorders Identification Test (AUDIT) from team and individual exercisers
Note. A Mann-Whitney $U$ test indicated that the two groups differed statistically significantly $(p=.021)$ from each other; the former had a greater mean rank (89.86) than the latter (72.93). 


\section{DISCUSSION}

The results of this work show that while the prevalence of alcohol use does not differ between team and individual exercisers, the severity of alcohol use-related problems appears to be greater in the former than the latter group. Indeed, the frequency of the clinical attention requiring cases was twice as high among team exercisers in contrast to individual exercisers. These findings are consistent with the results obtained with high school athletes (Kulesza et al., 2014), and expend those by showing that the differences between team and individual exercisers in alcohol use-related problems persist in the older adult stage as well. The results also agree with another earlier study, examining university students who participated in high-school athletics, in which statistically significant gender differences were disclosed between young men and women (Peretti-Watel, Beck, \& Legleye, 2002), and again, expend those findings to the adult exercisers. We could not compare our findings with studies directly investigating prevalence and level of problematic alcohol use in a group of heterogeneous physically active team and individual exercisers because we could not locate such studies.

Why did we not study a specific target group, such as people in a particular sport with constant exercise practice routines? There are several answers to this question. The first is that at the exploratory level, we need to assess whether the phenomena exists at all. While there were a few reports suggesting that sport participation (Kwan et al., 2014; Lisha \& Sussman, 2010) was linked to increased alcohol use problem, these reviews were conducted on research results obtained on adolescents and young adults. Further, direct or indirect studies of alcohol related problems in team and individual sports provide results that are restricted to adolescents and/or young adults (Kuleza et al., 2014; Peretti-Watel et al., 2002; Wichstrøm \& Wichstrøm, 2009). While science most often needs to be reductionist to answer specific questions, there are a few exemptions when looking at the global picture might determine if it is worth posing specific questions. Indeed, our results suggest that in a highly heterogeneous sample of exercisers, as based on the very wide ranges of exercise frequency and volume, which were unrelated to the AUDIT scores, we could detect differences in alcohol related problems between team and individual exercisers. Given that the results appear to be restricted to men, and that they could be replicated in the subsample of older (than studied to date) adults, we might assume that the results are due to a general tendency of males drinking more in regularly attended social settings, relatively independently of sport and exercise behaviour. This plausible hypothesis is not farfetched at all considering a recent comprehensive review showing that men use alcohol in social settings to regulate their uptight emotions (Sayette, 2017). After drinking in a social group, men become more talkative and smile with greater ease, which let the author conclude that alcohol as a social 'reward' of easingup and feeling good is a driving force behind heavy drinking socializing men. Therefore, stigmatizing sports or physical activity settings, simply because social encounters are regular and enduring over time, might be erroneous. However, it is possible that in some coherent teams the frequency of the social interactions and reasons to celebrate or moan (win or loss outcomes) may generate additional incentives for drinking.

The present exploratory findings show that independent of exercise parameters, in men only, there are greater alcohol use related problems if they exercise in team settings, and that the overall findings are consistent in older adults too. The results raise several new questions, while providing incentive for further research. The first is whether in regularly meeting teams or groups in a nonexercises or sport context similar findings would emerge. If yes, that would reinforce our conjecture and Sayette's theory that problematic alcohol use in social settings by men is a general, rather than an exercise or sport specific phenomenon. The second question then is, how comparable are the results obtained with heterogeneous exercisers, like the ones studied here, with those obtained with a cohesive sports team; any differences would require a look at the history, current frequency, emotional content, and level of interactions as the possible mediators of such differences.

Limitations. The current study is not without limitations. The first is that albeit the sample was randomly selected for the current inquiry, they came from a large dataset obtained from self-selected participants that poses an obvious, universal, and often irresolvable dilemma in most research, especially sensitive research with humans. Another limitation is that for determining prevalence we simply looked at use/no-use of alcohol within the past year in trying to identify drinkers and 
non-drinkers. Estimation of the units of alcohol could provide a clearer picture even though that may be subject to memory distortion and subjective bias. The sample size could also be criticized. However, this was not meant to be an epidemiological study and the needed sample size was calculated using $a$ priori; if these results were statistically significant with a presumably small sample, and on the bases of the low power non-parametric tests, one could only expect more robust findings in research with higher sample sizes. Further, the very heterogeneous nature (no delimitation for exercise volume and type, social background, etc.) of the current sample could be prone to criticism too. However, we were examining a general issue for which the heterogeneity served the purpose. Further studies may wish to address the new questions which emerged from the results of the current work in a specific manner.

\section{CONCLUSIONS}

The prevalence of alcohol use in team and individual exercises is similar. Team exercise participation is related to heightened alcohol use problems. Increased alcohol use problems in team exercises may only occur in men. Increased alcohol use problems in team exercises occur in adults as well. In general, men show more significant alcohol use problems than women.

Financial support. No funding was received for this work.

Ethical clearance. Ethical permission for the study was obtained from the Research Ethics Committee of the Faculty of Education and Psychology at ELTE Eötvös Loránd University.

Conflict of interest. The authors have no conflict of interest to declare.

\section{REFERENCES}

Babor, T. F., Higgins-Biddle, J. C., Saunders, J. B., \& Monteiro, M. G. (2001). AUDIT. The Alcohol Use Disorders Identification Test: Guidelines for use in primary care. 2nd ed. World Health Organization: Department for Mental Health \& Substance Dependence.

Becker, S. J., \& Curry, J. F. (2013). Testing the effects of peer socialization versus selection on alcohol and marijuana use among treated adolescents. Substance Use \& Misuse, 49(3), 234-242. doi: 10.3109/10826084.2013.824479

Black, D., Lawson, J., \& Fleishman, S. (1999). Excessive alcohol use by non-elite sportsmen. Drug and Alcohol Review, 18(2), 201-205. doi: 10.1080/09595239996644

Faul, F., Erdfelder, E., Buchner, A., \& Lang, A. G. (2009). Statistical power analyses using G*Power 3.1: Tests for correlation and regression analyses. Behavior Research Methods, 41(4), 1149-1160. doi: 10.3758/ brm.41.4.1149

Kulesza, M., Grossbard, J. R., Kilmer, J., Copeland, A. L., \& Larimer, M. E. (2014). Take one for the Team? Influence of team and individual sport participation on high school athlete substance use patterns. Journal of Child \& Adolescent Substance Abuse, 23(4), 217-223. doi:10.1080/1067828x.2013.786928

Kwan, M., Bobko, S., Faulkner, G., Donnelly, P., \& Cairney, J. (2014). Sport participation and alcohol and illicit drug use in adolescents and young adults: A systematic review of longitudinal studies.
Addictive Behaviors, 39(3), 497-506. doi: 10.1016/j. addbeh.2013.11.006

Lisha, N. E., \& Sussman, S. (2010). Relationship of high school and college sports participation with alcohol, tobacco, and illicit drug use: A review. Addictive Behaviors, 35(5), 399-407. doi: 10.1016/j. addbeh.2009.12.032

Peretti-Watel, P., Beck, F., \& Legleye, S. (2002). Beyond the U-curve: The relationship between sport and alcohol, cigarette and cannabis use in adolescents. Addiction, 97(6), 707-716. doi: 10.1046/j.1360-0443.2002.00116.x Sayette, M. A. (2017). The effects of alcohol on emotion in social drinkers. Behaviour Research and Therapy, 88 , 76-89. doi: 10.1016/j.brat.2016.06.005

Sønderlund, A. L., O’Brien, K., Kremer, P., Rowland, B., De Groot, F., Staiger, P., ... Miller, P. G. (2014). The association between sports participation, alcohol use and aggression and violence: A systematic review. Journal of Science and Medicine in Sport, 17(1), 2-7. doi: 10.1016/j.jsams.2013.03.011

Szabo, A., Griffiths, M. D., Aarhus Høglid, R., \& Demetrovics, Z. (2018). Drug, nicotine, and alcohol use among exercisers: Does substance addiction co-occur with exercise addiction? Addictive Behaviors Reports, 7, 26-31. doi: 10.1016/j.abrep.2017.12.001

Wichstrøm, T., \& Wichstrøm, L. (2009). Does sports participation during adolescence prevent later alcohol, tobacco and cannabis use? Addiction, 104(1), 138-149. doi: 10.1111/j.1360-0443.2008.02422.x
Corresponding author Attila Szabo Institute of Health Promotion and Sports Sciences Faculty of Education and Psychology ELTE Eötvös Loránd University

H-1117 Budapest, Bogdánfy u. 10 Hungary

Tel. +36702437123

Email szabo.attila@ppk.elte.hu 